\title{
GALLBLADDER TUBERCULOSIS MIMICKING CARCINOMA GALLBLADDER: A CASE REPORT
}

Chandra Shekhar ${ }^{1}$, Sanjay Kumar Bhat ${ }^{2}$, Mohammad Shuaib ${ }^{3}$, Imran Mohammad Ansari ${ }^{4}$, Vikas Tyagi ${ }^{5}$

\section{HOW TO CITE THIS ARTICLE:}

Chandra Shekhar, Sanjay Kumar Bhat, Mohammad Shuaib, Imran Mohammad Ansari, Vikas Tyagi. "Gallbladder Tuberculosis Mimicking Carcinoma Gallbladder: A Case Report". Journal of Evolution of Medical and Dental Sciences 2015; Vol. 4, Issue 65, August 13; Page: 11428-11431, DOI: 10.14260/jemds/2015/1649

ABSTRACT: Gallbladder tuberculosis (GT) is a very rare and curable disease but sometimes it can be confused with other gallbladder diseases like gallbladder carcinoma. Very few cases have been reported in the medical literature. The first case of GT was described in 1870 by Gaucher. A correct preoperative diagnosis of GT is unexpected. PRESENTATION OF CASE: A patient underwent surgery with a preoperative diagnosis of gallbladder carcinoma after ultrasonography (USG) and contrast enhanced computed tomography (CECT) scan after histopathological examination the disease turned out to be gallbladder tuberculosis. DISCUSSION: The gallbladder is highly resistant to tuberculous infection, and the presence of cholelithiasis and cystic duct obstruction is essential for the development of GT.[1,2] About 70\% of GT cases are accompanied by gallstones.[2] GT often occurs in association with other intra-abdominal tuberculosis, and usually in women over 30 years of age.[2] $\mathrm{A}$ wide spectrum of symptoms have been described in patients with GT, including abdominal pain in right hypochondrium, weight loss, fever, anorexia, diarrhea, nausea, vomiting and a palpable abdominal mass.[2] A correct diagnosis of GT is difficult, and it is usually made after cholecystectomy.[1] The differential diagnosis of GT includes acute and chronic cholecystitis, polypoid lesions and gallbladder carcinoma.[2] CONCLUSION: Gallbladder tuberculosis can mimic gallbladder carcinoma.

KEYWORDS: Contrast Enhanced Computed Tomography, Gallbladder, Tuberculosis, Gallbladder Cancer.

INTRODUCTION: Abdominal tuberculosis is common in developing countries but gallbladder tuberculosis (GT) is extremely rare.[1,2] Only very few cases have been reported in the medical literature.[1] A correct preoperative diagnosis of GT is unusual, and the condition is frequently confused with other gallbladder diseases like gallbladder carcinoma.

PRESENTATION OF CASE: A 32-year-old woman was admitted into our department for occasional right upper abdominal pain, weight loss, decreased appetite, off and on fever. Abdominal examination showed a palpable non-tender gallbladder. Laboratory tests were normal except for mild anaemia (Hb-8.2gm). On ultrasonogram (USG) a thick walled gallbladder, cholelithiasis, portal lymphadenopathy and minimal fluid in the pouch of Douglas were detected. An abdominal contrast enhanced computed tomography (CECT) scan showed a thick walled gallbladder, a single large stone (Approx. $2.5 \mathrm{~cm}$ ) and portal lymphadenopathy. A diagnosis of gallbladder carcinoma was made (Figure 1).

Diagnostic laparoscopy showed thick walled gallbladder, enlarged cystic and portal lymph nodes and straw coloured fluid in the pouch of Douglas. Biopsy of gallbladder tissue and intraoperative frozen section showed features consistent with tuberculosis. The operation was converted into open surgery and cholecystectomy and cystic and portal lymphadenectomy were 
carried out the postoperative period was uneventful. Definitive histological study of the gallbladder, cystic and portal lymph nodes showed granulomatous reaction with multinucleated giant cells and caseous necrosis which were compatible with GT.

Postoperatively, a Mantoux test was negative. A chest X-ray showed no signs of tuberculous infection. Antituberculous drugs were given for six months [2HRZE+4HR].

DISCUSSION: The gallbladder is highly resistant to tuberculous infection, and the presence of cholelithiasis and cystic duct obstruction is essential for the development of GT.[1,2] About 70\% of GT cases are accompanied by gallstones.[2] In a study of 280 patients with hepatobiliary tuberculosis, only one patient had gallbladder involvement.[3] In their experience of over two decades with gallbladder and pancreatic tuberculosis, Saluja et al.[4] found only three cases of tuberculous gallbladder involvement. The rarity of tuberculosis involving gallbladder could be attributed to the fact that bile has an inhibitory effect on gallbladder mucosa for development of tuberculosis. Four types of gallbladder tuberculosis (GBTB) have been described: A) Miliary TB in children with ulcerating tubercles in the gallbladder. B) GBTB in association with severe generalized TB. C) Isolated GBTB. D) Gallbladder involvement in association with TB in other intraperitoneal organs.[5] The fourth group is said to be the commonest type.[6] Our case belonged to the third type of isolated gallbladder TB. The routes of infection in GBTB can be canalicular, lymphatic or hematogenous.[1],[5]

GT often occurs in association with other intra-abdominal tuberculosis, and usually in women over 30 years of age.[2] Histologically, the lesion may be in the form of a localized ulceration or there may be typical tuberculous nodules of varied sizes and numbers in the gallbladder wall.

A wide spectrum of symptoms have been described in patients with GT, including abdominal pain in right hypochondrium, weight loss, fever, anorexia, diarrhea, nausea, vomiting and a palpable abdominal mass. ${ }^{[2]}$ Anemia, elevated eosinophil sedimentation rate (ESR) and positive tuberculin test are usually found on laboratory tests.

The correct diagnosis of GT is difficult, and it is usually made after cholecystectomy.[1] Ultrasound and CT may show an enlarged gallbladder, with a thickened gallbladder wall, soft tissue masses, or nodular lesions, although neither ultrasound nor CT lesions are specific.[2] The diagnosis of tuberculosis in other abdominal organs (Liver, spleen, lymph nodes or peritoneum) is very helpful in diagnosing GT.

The differential diagnosis of GT includes acute and chronic cholecystitis, polypoid lesions and gallbladder carcinoma.[2] The presence of a mass that fills the gallbladder containing cholelithiasis is indistinguishable from gallbladder carcinoma.[1] Moreover, both GT and carcinoma can give rise to enlarged regional lymph nodes. The presence of liver metastasis or liver infiltration suggests gallbladder carcinoma. On the other hand, lung lesions or mesenteric thickening is more frequently seen in patients with tuberculous infection.

CONCLUSION: Gallbladder tuberculosis can mimic gallbladder carcinoma which can lead to difficulties in the management of:

1. The similar radiological and clinical features of gallbladder carcinoma and gallbladder tuberculosis can lead to a delay in diagnosis and treatment of gallbladder tuberculosis which is potentially curable. 


\section{CASE REPORT}

2. Tuberculous enlargement of the cystic lymph node can cause the gallbladder to become distended and palpable and present with cholecystitis.

3. A suspected case of gallbladder carcinoma should be confirmed by fine needle aspiration cytology even if the tumor appears to be at an advanced stage.

\section{REFERENCES:}

1. Jain R, Sawhney S, Bhargava D, Berry M. Gallbladder tuberculosis: sonographic appearance. J Clin Ultrasound. 1995; 23: 327-329.

2. Yu R, Liu Y. Gallbladder tuberculosis: case report. Chin Med J (Engl). 2002; 115: 1259-1261

3. Amarapurkar DN, Patel ND, Amarapurkar AD. Hepatobiliary tuberculosis in western India. Indian J Pathol Microbiol. 2008; 51: 175-181.

4. Saluja SS, Ray S, Pal S, et al. Hepatobiliary and pancreatic tuberculosis: A two decade experience. BMC Surg. 2007; 24: 7-10.

5. Bergdahl L, Boquist L. Tuberculosis of gallbladder. Br. J Surg 1972; 59: 289-292.

6. Abasca J, Martin F, Abreu I et al. Atypical hepatic tuberculosis presenting as obstructive jaundice. Am J Gastro Enterol 1985; 83: 1183-1186.

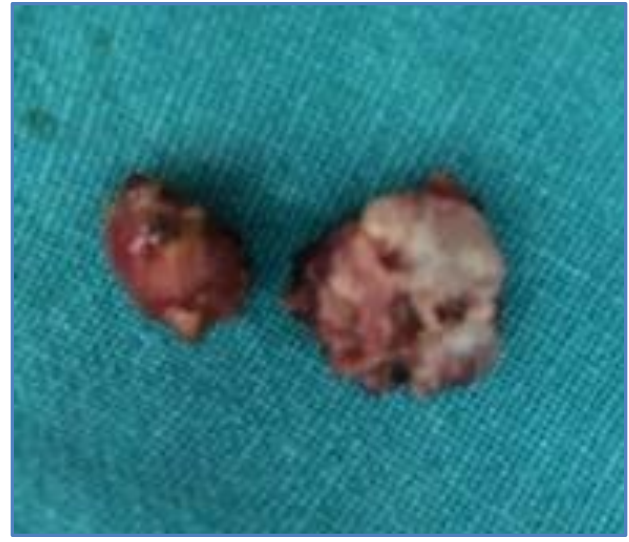

Cystic and Protal Lymphnode

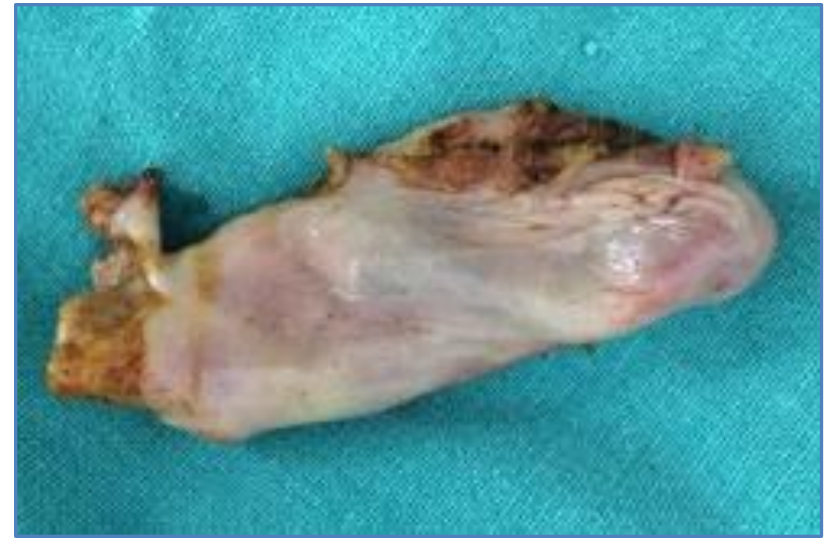

Gallbladder Specimen

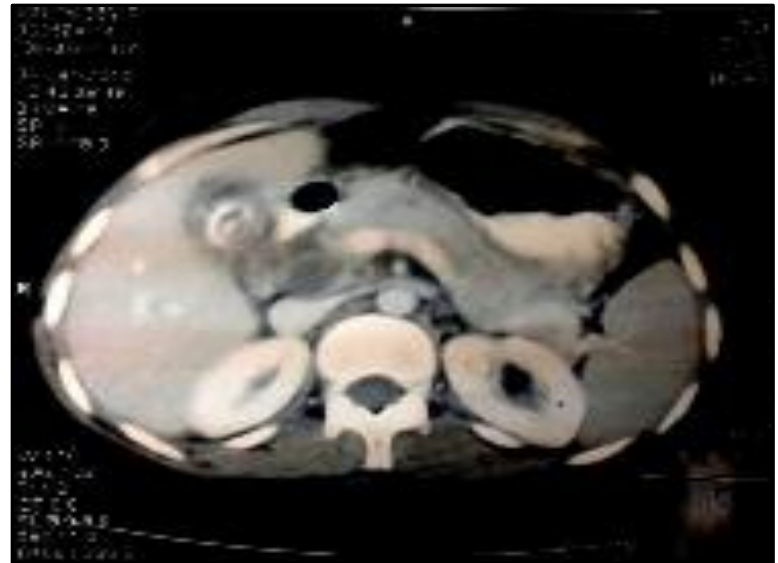

Thick walled gallbladder with single large stone (Approx. 2.5cm) with portal lymphadenopathy 


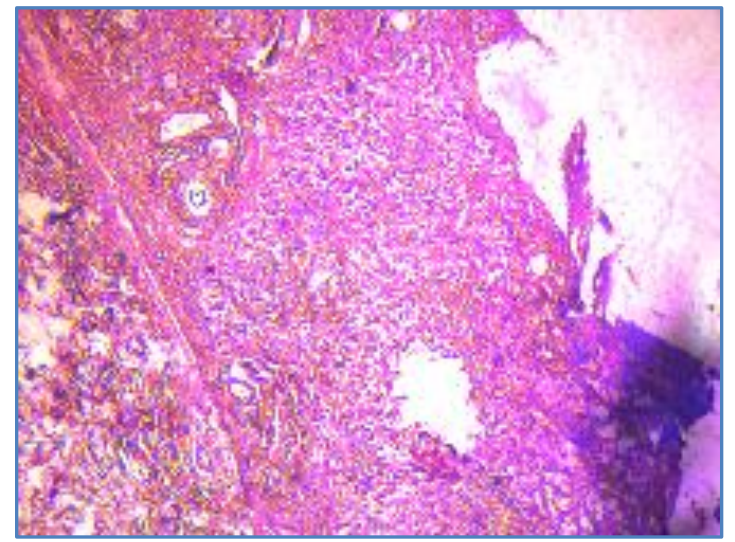

\section{AUTHORS:}
1. Chandra Shekhar
2. Sanjay Kumar Bhat
3. Mohammad Shuaib
4. Imran Mohammad Ansari
5. Vikas Tyagi

\section{PARTICULARS OF CONTRIBUTORS:}

1. Assistant Professor, Department of General Surgery, Era Medical College, Lucknow.

2. Associate Professor. Department of general surgery, Era medical college, Lucknow.

3. Junior Resident, Department of General Surgery, Era Medical College, Lucknow.

\section{FINANCIAL OR OTHER} COMPETING INTERESTS: None
4. Senior Resident, Department of General Surgery, Integral University.

5. Junior Resident, Department of General Surgery, Era Medical College, Lucknow.

\section{NAME ADDRESS EMAIL ID OF THE CORRESPONDING AUTHOR:}

Chandra Shekhar,

House No. 1898,

E Block, Rajajipuram,

Lucknow.

E-mail: csr13582@gmail.com

Date of Submission: 22/06/2015.

Date of Peer Review: 23/06/2015.

Date of Acceptance: 07/08/2015.

Date of Publishing: 13/08/2015. 